\title{
The Influence of Socioeconomic Factors on Quality-of-Life After Laparoscopic Gastric Bypass Surgery
}

\author{
Karin Gryth $^{1} \cdot$ Carina Persson ${ }^{2,3} \cdot$ Ingmar Näslund $^{1} \cdot$ Magnus Sundbom $^{4} \cdot$ Erik Näslund $^{5} \cdot$ Erik Stenberg $^{1}$ (D)
}

Published online: 12 June 2019

(C) The Author(s) 2019

\begin{abstract}
Introduction Patients with low socioeconomic status have been reported to experience poorer outcome after several types of surgery. The influence of socioeconomic factors on health-related quality-of-life (HRQoL) after bariatric surgery is unclear. Materials and Methods Patients operated with a primary laparoscopic gastric bypass procedure in Sweden between 2007 and 2015 were identified in the Scandinavian Obesity Surgery Register. Patients with a completed assessment of health-related quality-of-life based on the Obesity-related Problem Scale (OP Scale) were included in the study. Socioeconomic status was based on data from Statistics Sweden.

Results A total of 13,723 patients ( $32 \%$ of the 43,096 operated during the same period), with complete OP scores at baseline and two years after surgery, were included in the study. Age, lower preoperative BMI, male gender, higher education, professional status and disposable income as well as not receiving social benefits (not including retirement pension), and not a first- or secondgeneration immigrant, were associated with a higher postoperative HRQoL. Patients aged 30-60 years, with lower BMI, higher socioeconomic status, women and those born in Sweden by Swedish parents experienced a higher degree of improvement in HRQoL. Postoperative weight-loss was associated with higher HRQoL (unadjusted $B$ 16.3, 95\%CI 14.72-17.93, $p<0.0001$ ). Conclusion At 2 years, a strong association between weight loss and improvement in HRQoL was seen, though several factors influenced the degree of improvement. Age, sex, preoperative BMI and socioeconomic status all influence the postoperative HRQoL as well as the improvement in HRQoL after laparoscopic gastric bypass surgery.
\end{abstract}

Keywords Bariatric surgery $\cdot$ Gastric bypass $\cdot$ Laparoscopy $\cdot$ Quality-of-life $\cdot$ Risk factors

\section{Introduction}

Obesity is associated with increased risk for multiple metabolic and cardiovascular sequelae [1,2], cancer [3] and shorter life expectancy [4]. Bariatric surgery reduces the incidence of

Erik Stenberg

erik.stenberg@regionorebrolan.se

1 Department of Surgery, Faculty of Medicine and Health, Örebro University Hospital, SE-701 85 Örebro, Sweden

2 Department of Community Medicine and Public Health, Faculty of Medicine and Health, Örebro University, Örebro, Sweden

3 Department for Sustainable Development, Region Örebro County, Örebro, Sweden

4 Department of Surgical Sciences, Uppsala University, Uppsala, Sweden

5 Division of Surgery, Department of Clinical Sciences, Danderyd Hospital, Karolinska Institutet, Stockholm, Sweden these sequelae, new cancer development and the risk for premature death in individuals with BMI $>35 \mathrm{~kg} / \mathrm{m}^{2}$ [5-8].

Living with severe obesity is also associated with several negative psychological consequences, body image concerns, low self-esteem and low health-related quality-of-life (HRQoL) $[9,10]$. HRQoL is a multidimensional concept including physical/somatic, psychosocial/mental and social aspects. Different instruments can be used to estimate HRQoL, for example, the Obesity-related Problem Scale (OP Scale), focusing on the psychosocial burden of the disease [11]. HRQoL is known to improve after bariatric surgery, especially in men, younger patients and patients with satisfactory weight loss $[12,13]$.

Access to bariatric surgery for treatment of severe obesity is not equal based on socioeconomic status [14, 15]. Furthermore, patients with lower socioeconomic status and low level of education have an increased risk for short-term complications after bariatric surgery [16] and poorer outcome after other surgical procedures such as arthroplasty and 
neurosurgery $[17,18]$. Whether socioeconomic status and education level influence HRQoL after bariatric surgery remains unclear.

The aim of the present study was to investigate to what extent socioeconomic status influences HRQoL after laparoscopic gastric bypass surgery in a large nationwide cohort.

\section{Methods}

All patients operated with primary laparoscopic gastric bypass surgery in Sweden between June 1, 2007 and July 31, 2015, were identified in the Scandinavian Obesity Surgery Register (SOReg). Missing or incomplete registration of HRQoL estimates, and age $<18$ years were exclusion criteria. Baseline characteristics, quality-of-life estimates and follow-up data were based on data from the SOReg. Data on socioeconomic factors (education, profession, disposable income, residence, marital status, social benefits and heritage) were based on data from Statistics Sweden.

Comorbidity at baseline was defined as pharmacological treatment or continuous positive airway pressure treatment (in the case of sleep apnoea) for at least one of six specified obesity-related comorbidities (sleep apnoea, hypertension, type 2 diabetes, dyslipidaemia, dyspepsia/GERD and depression). Socioeconomic factors were subdivided into groups based on the International Standard Classification of Occupations from 1988 (ISCO-88) for profession, the Swedish Association of Local Authorities and Regions' definitions for residence and accepted standards for other variables.

\section{Procedure}

The surgical technique for laparoscopic gastric bypass surgery is basically standard throughout Sweden with $99 \%$ using the antecolic, antegastric laparoscopic gastric bypass procedure (Lönroth technique) [19], with a Roux limb of approximately $100 \mathrm{~cm}$, and a biliopancreatic limb of $50 \mathrm{~cm}$ as standard.

\section{Outcomes}

Main outcome measure was improvement in health-related quality-of-life, estimated with the Obesity-related Problem Scale (OP Scale). The OP Scale is a disease-specific scale measuring the impact of obesity on psychosocial functioning, previously validated for patients undergoing bariatric surgery [11]. The scale consists of eight questions, on common obesity-related problems, aggregated into a score from 0 to 100 with lower scores representing better HRQoL [11].

\section{Statistics}

Quality-of-life was analysed as mean difference between baseline value and reported estimate at two years, using unadjusted linear regression (only adjusted for baseline OPscore) and adjusted linear regression adjusted for baseline OP-score, excess BMI loss at two years $(\% \mathrm{EBMIL}=($ Initial BMI - BMI two years after surgery)/(Initial BMI - 25)), age, sex, presence of sleep apnoea, hypertension, diabetes, dyslipidaemia, dyspepsia/GERD and depression.

Due to the multiplicity of variables analysed, the Bonferroni-Holm method was used to compensate for multiple calculations [20].

Sensitivity analyses of patients excluded were made using logistic regression for categorical variables and linear regression for continuous variables.

$P<0.05$ was considered to be statistically significant.

IBM SPSS version 25 was used for all statistical analyses.

\section{Ethics}

The study was approved by the Stockholm Regional Ethics committee and was conducted in accordance with the ethical standards of the 1964 Helsinki Declaration and its later amendments.

\section{Results}

During the inclusion period, 43,096 patients operated with a primary laparoscopic gastric bypass procedure were identified. After exclusion of patients without registered baseline quality-of-life estimates $(n=11,245)$, incomplete baseline quality-of-life estimates $(n=173)$, non-registered quality-oflife estimates at two years $(n=17,923)$ and incomplete quality-of-life estimates at two years $(n=32) ; 13,723$ patients $(32 \%)$ remained within the study group.

Baseline characteristics of the study group are presented in Table 1 . Sensitivity analysis of the excluded patients revealed younger age ( 40.2 vs. 42.1 years, $p<0.001)$, lower prevalence of hypertension $(24.1 \%$ vs. $27.2 \%, p<0.001)$, higher prevalence of depression $(15.1 \%$ vs. $13.3 \%, p<0.001)$, larger proportion of patients with lower disposable income $(<20$ th percentile $28.9 \%$ vs. $22.8 \%, 20-50$ th percentile $32.4 \%$ vs. $31.9 \%$, $p<0.001$ ), small differences in marital status (married/partner $41.9 \%$ vs. $45.1 \%$, reference, divorced/widow/widower $16.1 \%$ vs. $15.4 \%, p<0.001$, single $42.0 \%$ vs. $39.5 \%, p<0.001$ ), higher proportion receiving disability pension/early retirement $(12.9 \%$, vs. $11.9 \%, p<0.001)$ or social benefits $(8.8 \%$ vs. $4.9 \%, p<0.001)$ and higher proportion of patients being born outside of Sweden by non-Swedish parents (15.8\% vs. $12.5 \%, p<0.001)$. 
Table 1 Baseline characteristics

Missing data

$\mathrm{BMI}$, mean $\pm \mathrm{SD}\left(\mathrm{kg} / \mathrm{m}^{2}\right)$

Age, mean $\pm \mathrm{SD}$ (years)

Comorbidity, $n(\%)$

Sleep apnoea, $n(\%)$

Hypertension, $n(\%)$

Diabetes, $n(\%)$

Dyslipidaemia, $n(\%)$

Dyspepsia/GERD, $n(\%)$

Depression, $n(\%)$

Smoking

None

Previous smoking

Active smoking

Education

Primary education $<9$ years

Secondary education

Higher education $<3$ years

Higher education $>3$ years

Profession

Senior officials and management

Professionals and technicians

Clerical support workers

Services and sales workers

Manual labour

Elementary occupation

Disposable income

$<20$ th percentile

20-50th percentile

50-80th percentile

$>80$ th percentile

Residence

Large city and municipality

Medium-sized town and municipality

Small town, urban area, rural municipality

Marital status

Married/partner

Divorced/widow/widower

Single

Financial aid

None

Retirement pension

Disability pension/early retirement

Social benefits

Heritage

Swedish-born, Swedish-descendant

Swedish-born, non-Swedish-descendant

Born outside Sweden
$0(0.0 \%)$

$0(0.0 \%)$

$0(0.0 \%)$

$42.1 \pm 5.28$

$42.2 \pm 11.07$

$6992(51.0 \%)$

$1404(10.2 \%)$

$3738(27.2 \%)$

$1968(14.3 \%)$

$1395(10.2 \%)$

$1233(9.0 \%)$

$1826(13.3 \%)$

$3930(28.6 \%)$

$6664(68.0 \%)$

$1928(19.7 \%)$

$1201(12.3 \%)$

$51(0.4 \%)$

$2152(15.7 \%)$

$8364(61.2 \%)$

$1561(11.4 \%)$

1595 (11.7\%)

$1884(13.7 \%)$

$512(4.3 \%)$

$2926(24.7 \%)$

$1297(11.0 \%)$

$4469(37.7 \%)$

1805 (15.2\%)

$830(7.0 \%)$

$141(1.0 \%)$

3095 (22.8\%)

4337 (31.9\%)

$4472(32.9 \%)$

$1678(12.4 \%)$

$18(0.1 \%)$

5011 (36.6\%)

$4618(33.7 \%)$

4076 (29.7\%)

$13(0.1 \%)$

$6184(45.1 \%)$

$2108(15.4 \%)$

$5418(39.5 \%)$

$0(0.0 \%)$

$11,188(81.5 \%)$

$232(1.7 \%)$

1635 (11.9\%)

$668(4.9 \%)$

$15(0.1 \%)$

$11,319(82.6 \%)$

674 (4.9\%)

$1715(12.5 \%)$ 
Data on weight loss was available for 13,481 patients at two years after surgery. The average BMI-loss at two years was $13.6 \pm 4.3$ BMI units, average percentage total weight loss (\%TWL) was $32.1 \pm 8.6 \%$, percentage excess BMI-loss (\%EBMIL) $82.5 \pm 24.0 \%$.

Mean OP-score at baseline, $61.6 \pm 26.3$, had improved to $20.7 \pm 24.22$ years after surgery $(p<0.001)$.

Younger patients and women reported worse OP-scores in the preoperative setting. Higher work status and manual labour, higher disposable income, being married/partner, not receiving social benefits (not including retirement pension) and being born in Sweden by Swedish parents were associated with better preoperative OP-scores (Table 2). At two years after surgery, higher age, lower BMI, male gender, higher education, higher work status (except for manual labour), higher disposable income, being married/partner, not receiving social benefits and being born in Sweden by Swedish parents were associated with better OP-scores (Table 2).

Better improvements in OP-score were seen amongst patients aged 30-60 years, with lower BMI, women, as well as those with higher education, higher work status, residents of small towns, married/partners, not receiving social benefits and born in Sweden by Swedish parents (Table 2).

There was a strong association between weight loss (\%EBMIL) at two years and improvement in OP-score (unadjusted B 16.3, 95\%CI 14.72-17.93, $p<0.0001)$. After adjustment for year of surgery, age, BMI, comorbid disease and $\%$ EBMIL, the following factors strongly influenced the effect of bariatric surgery on disease-specific health-related qualityof-life: level of education; disposable income; marital status; social benefits or not; and heritage. Place of residence and profession had less impact on the results (Table 3 ).

\section{Discussion}

Younger patients with higher BMI, single or divorced, women, first- or second-generation immigrants and patients with lower work status (except for manual labour) and lower income reported poorer preoperative health-related quality-oflife. All groups experienced significant improvement after gastric bypass surgery. However, age, sex, BMI, education and work status as well as income, receiving social benefits, marital status and ethnicity all influenced the degree of improvement.

Health-related quality-of-life prior to surgery increased with age, a finding consistent with previous reports that younger people with morbid obesity estimate their overall health and physical function as being lower than their older counterparts $[21,22]$. The higher degree of improvement postoperatively amongst patients aged $30-60$ years tended to nullify this difference two years after surgery, whereas patients younger than 30 years experienced less improvement two years after surgery. This difference may be the result of differences in self-image and expectations of bariatric surgery amongst younger patients. Whilst health concerns appear to be a strong motivation for weight loss amongst older patients, younger patients are more often motivated by appearance and social factors [23]. A higher focus on aesthetic appearance and factors not related to health benefits may increase the risk for disappointment with the postoperative result. Intimidation, discrimination and other negative social consequences of obesity frequently occur, and this problem appears to be particularly great amongst younger women [24]. Women with obesity generally report lower self-esteem [25], and the association between gender and HRQoL has been reported previously [26]. In the present study, women experienced greater improvement in their HRQoL after surgery, although they still reported a somewhat lower HRQoL compared with men two years after surgery.

BMI itself is strongly related to improvement in HRQoL $[13,21,26]$. The strong association between preoperative BMI and postoperative HRQoL are well in line with previous studies $[10,13]$. Although heavier patients (in particular younger patients) tend to lose more weight after bariatric surgery, they still have difficulties reaching a BMI < 30 [27].

In most societies, the level of education gives a good estimate of socioeconomic status. Before surgery, patients with less than nine-year education reported a slightly lower HRQoL. However, after surgery, patients with higher education improved more than patients with lower education. Although the difference is likely to be multifactorial, one contributing factor may be differences in health literacy (the ability to understand access and use information to make decisions about their health). Although higher education itself does not always lead to high health literacy, a lower level of education is strongly associated with lower health literacy [28, 29].

In general, patients with higher professional status reported better HRQoL before and after surgery. An interesting exception to this is the group "manual labourers", who reported better HRQoL both before and after surgery. Due to the nature of their work, this group is likely to have a higher level of daily physical activity and less often a sedentary lifestyle, both factors influencing HRQoL [30]. Furthermore, a higher income was associated with a higher HRQoL prior to surgery, a difference that became even greater after surgery. Both higher professional status and higher income were thus positive factors for improvement in HRQoL after bariatric surgery. This is well in line with a previous study from ten countries stating that level of education and income are clearly related to selfassessed health [31]. Furthermore, low income may be a barrier to effective postoperative weight loss [32], which in part may contribute to the lower postoperative HRQoL amongst patients with lower income. Patients taking early retirement, disability pension or requirement of social benefits also experience lower HRQoL before and after surgery. In a previous study by Raoof et al., being out of work (due to sick leave, 
Table 2 Health-related quality-of-life at baseline and 2 years after surgery

\begin{tabular}{|c|c|c|c|c|c|}
\hline & $\mathrm{N}$ & Base-line OP-score & OP-score at 2 years & Mean difference & Unadjusted $P^{\mathrm{a}}$ \\
\hline \multicolumn{6}{|l|}{ Age } \\
\hline$<30$ & 2015 & $67.9 \pm 24.30$ & $30.0 \pm 29.93$ & $37.9 \pm 31.38$ & Reference \\
\hline $30-40$ & 3445 & $65.2 \pm 29.94 *$ & $21.7 \pm 23.98 *$ & $43.5 \pm 29.79$ & $<0.0001 *$ \\
\hline $40-50$ & 4519 & $60.1 \pm 46.48^{*}$ & $18.6 \pm 23.07 *$ & $41.4 \pm 29.88$ & $<0.0001 *$ \\
\hline $50-60$ & 2984 & $57.6 \pm 26.77 *$ & $17.5 \pm 22.95^{*}$ & $40.1 \pm 29.02$ & $<0.0001^{*}$ \\
\hline$>60$ & 760 & $52.2 \pm 28.25^{*}$ & $16.5 \pm 22.34 *$ & $35.7 \pm 29.61$ & $<0.0001 *$ \\
\hline \multicolumn{6}{|l|}{ BMI } \\
\hline$<40$ & 5192 & $61.8 \pm 25.95$ & $17.9 \pm 22.95$ & $43.8 \pm 29.84$ & Reference \\
\hline $40-50$ & 7504 & $61.3 \pm 26.60$ & $21.8 \pm 24.50 *$ & $39.6 \pm 29.77$ & $<0.0001 *$ \\
\hline $50-60$ & 945 & $61.2 \pm 26.07$ & $27.0 \pm 26.39^{*}$ & $34.7 \pm 30.56$ & $<0.0001 *$ \\
\hline$>60$ & 82 & $64.6 \pm 23.87$ & $29.7 \pm 25.31 *$ & $34.8 \pm 28.01$ & $<0.0001 *$ \\
\hline \multicolumn{6}{|l|}{ Sex } \\
\hline Female & 10,556 & $65.2 \pm 24.95$ & $22.0 \pm 24.67$ & $43.2 \pm 29.82$ & Reference \\
\hline Male & 3167 & $49.3 \pm 26.98 *$ & $16.4 \pm 22.07 *$ & $32.9 \pm 29.96$ & $0.005^{*}$ \\
\hline \multicolumn{6}{|l|}{ Education } \\
\hline Primary education $<9$ years & 2152 & $62.9 \pm 27.43$ & $25.5 \pm 27.43 *$ & $37.3 \pm 31.91$ & $<0.0001^{*}$ \\
\hline Secondary education & 8364 & $61.3 \pm 26.39$ & $20.7 \pm 24.02$ & $40.6 \pm 29.98$ & Reference \\
\hline Higher education $<3$ years & 1561 & $61.1 \pm 25.65$ & $17.8 \pm 21.87 *$ & $43.3 \pm 27.95$ & $<0.0001^{*}$ \\
\hline Higher education $>3$ years & 1595 & $61.2 \pm 24.80$ & $16.8 \pm 21.02 *$ & $44.4 \pm 29.05$ & $<0.0001 *$ \\
\hline \multicolumn{6}{|l|}{ Profession } \\
\hline Senior officials and management & 512 & $54.2 \pm 27.08^{*}$ & $10.8 \pm 16.51^{*}$ & $43.4 \pm 28.28$ & $<0.0001^{*}$ \\
\hline Professionals and technicians & 2926 & $59.9 \pm 25.76^{*}$ & $15.7 \pm 20.34^{*}$ & $44.1 \pm 27.75$ & $<0.0001 *$ \\
\hline Clerical support workers & 1297 & $60.9 \pm 25.67 *$ & $19.7 \pm 23.13 *$ & $41.3 \pm 28.72$ & 0.013 \\
\hline Services and sales workers & 4469 & $64.9 \pm 25.06$ & $22.5 \pm 24.69$ & $42.4 \pm 30.67$ & Reference \\
\hline Manual labour & 1805 & $54.4 \pm 27.46^{*}$ & $17.2 \pm 21.86^{*}$ & $37.2 \pm 29.01$ & $<0.0001 *$ \\
\hline Elementary occupation & 830 & $62.4 \pm 26.52$ & $23.8 \pm 25.82$ & $38.6 \pm 31.68$ & 0.039 \\
\hline \multicolumn{6}{|l|}{ Disposable income } \\
\hline$<20$ th percentile & 3095 & $65.8 \pm 26.12$ & $29.6 \pm 27.52$ & $36.2 \pm 31.66$ & Reference \\
\hline $20-50$ th percentile & 4337 & $63.6 \pm 25.46^{*}$ & $22.0 \pm 24.51 *$ & $41.6 \pm 30.11$ & $<0.0001^{*}$ \\
\hline $50-80$ th percentile & 4472 & $59.1 \pm 26.30^{*}$ & $16.5 \pm 21.11 *$ & $42.5 \pm 29.24$ & $<0.0001 *$ \\
\hline$>$ 80th percentile & 1678 & $55.5 \pm 26.79 *$ & $12.5 \pm 18.76^{*}$ & $42.9 \pm 27.39$ & $<0.0001 *$ \\
\hline \multicolumn{6}{|l|}{ Residence } \\
\hline Large city and municipality & 5011 & $61.1 \pm 26.40$ & $21.3 \pm 24.69$ & $39.7 \pm 30.18$ & Reference \\
\hline Medium-sized town and municipality & 4618 & $61.9 \pm 26.53$ & $20.7 \pm 23.94$ & $41.2 \pm 30.12$ & 0.066 \\
\hline Small town, urban area, rural municipality & 4076 & $61.7 \pm 25.92$ & $20.0 \pm 23.87 *$ & $41.7 \pm 29.46$ & $0.001 *$ \\
\hline \multicolumn{6}{|l|}{ Marital status } \\
\hline Married/partner & 6184 & $60.0 \pm 26.40$ & $17.4 \pm 22.46$ & $42.6 \pm 29.60$ & Reference \\
\hline Divorced/widow/widower & 2108 & $63.0 \pm 26.42 *$ & $22.3 \pm 25.46^{*}$ & $40.7 \pm 30.78$ & $<0.0001 *$ \\
\hline Single & 5418 & $62.7 \pm 26.06^{*}$ & $23.9 \pm 25.11 *$ & $38.8 \pm 29.91$ & $<0.0001 *$ \\
\hline \multicolumn{6}{|l|}{ Financial aid } \\
\hline None & 11,188 & $60.9 \pm 26.11$ & $18.7 \pm 22.58$ & $42.3 \pm 29.30$ & Reference \\
\hline Retirement pension & 232 & $50.0 \pm 27.54^{*}$ & $15.4 \pm 20.74$ & $34.5 \pm 28.07$ & 0.705 \\
\hline Disability pension/early retirement & 1635 & $64.1 \pm 29.93 *$ & $28.7 \pm 28.32 *$ & $35.4 \pm 31.92$ & $<0.0001 *$ \\
\hline Social benefits & 668 & $69.6 \pm 25.06^{*}$ & $37.6 \pm 29.51 *$ & $32.0 \pm 33.12$ & $<0.0001^{*}$ \\
\hline \multicolumn{6}{|l|}{ Heritage } \\
\hline Swedish-born, Swedish-descendant & 11,319 & $61.0 \pm 26.24$ & $19.3 \pm 23.23$ & $41.7 \pm 29.33$ & Reference \\
\hline Swedish-born, non-Swedish-descendant & 674 & $62.7 \pm 26.67$ & $24.1 \pm 26.49^{*}$ & $38.6 \pm 30.57$ & $<0.0001^{*}$ \\
\hline Born outside Sweden & 1715 & $64.6 \pm 26.35^{*}$ & $28.7 \pm 27.64 *$ & $35.9 \pm 33.14$ & $<0.0001^{*}$ \\
\hline
\end{tabular}

${ }^{\text {a }}$ Adjusted for baseline OP-score

* Significant $p$ value $(p<0.05)$ after correction for multiple calculations

retirement or unemployment) was associated with less improvement in HRQoL after bariatric surgery [13]. Besides the obvious economic stress of not being employed, the loss of social support and social relationships established at work may also contribute to this difference in self-reported quality of life [33]. The social support of partnership/marriage is linked to higher subjective quality of life [34] and may well explain the higher HRQoL reported by patients in a relationship/marriage situation at the time of surgery. Despite challenges to the relationship that many couples experience after bariatric surgery [35], a shared economy may cause less economic stress than that experienced by singles, divorced, widows or widowers who generally have a lower disposable income.

First- and second-generation immigrants had a lower self-reported HRQoL than patients born in Sweden by Swedish parents. Furthermore, this group reported significantly less improvement after surgery. This 
Table 3 Standardized coefficients for the improvement in health-related quality-of-life 2 years after surgery

\begin{tabular}{|c|c|c|}
\hline & Adjusted $B(95 \% \mathrm{CI})$ & Adjusted $P^{\mathrm{a}}$ \\
\hline \multicolumn{3}{|l|}{ Education } \\
\hline Primary education $<9$ years & Reference & Reference \\
\hline Secondary education & $4.70(3.60-5.80)$ & $<0.0001^{*}$ \\
\hline Higher education $<3$ years & $2.70(1.50-3.89)$ & $<0.0001 *$ \\
\hline Higher education $>3$ years & $1.85(1.29-2.44)$ & $<0.0001 *$ \\
\hline \multicolumn{3}{|l|}{ Profession } \\
\hline Senior officials and management & $2.15(1.40-2.90)$ & $<0.0001 *$ \\
\hline Professionals and technicians & $2.20(1.67-2.73)$ & $<0.0001 *$ \\
\hline Clerical support workers & $0.86(-0.59-2.31)$ & 0.243 \\
\hline Services and sales workers & Reference & Reference \\
\hline Manual labour & $0.83(-0.77-2.43)$ & 0.311 \\
\hline Elementary occupation & $-1.21(-2.10--0.31)$ & $0.008 *$ \\
\hline \multicolumn{3}{|l|}{ Disposable income } \\
\hline$<20$ th percentile & Reference & Reference \\
\hline 20-50th percentile & $5.72(4.58-6.87)$ & $<0.0001 *$ \\
\hline 50-80th percentile & $4.51(3.96-5.06)$ & $<0.0001 *$ \\
\hline$>80$ th percentile & $3.65(3.14-4.16)$ & $<0.0001 *$ \\
\hline \multicolumn{3}{|l|}{ Residence } \\
\hline Large city and municipality & Reference & Reference \\
\hline Medium-sized town and municipality & $1.23(0.31-2.14)$ & $0.009^{*}$ \\
\hline Small town, urban area, rural municipality & $1.03(0.55-1.50)$ & $<0.0001^{*}$ \\
\hline \multicolumn{3}{|l|}{ Marital status } \\
\hline Married/partner & Reference & Reference \\
\hline Divorced/widow/widower & $-4.64(-5.75--3.55)$ & $<0.0001 *$ \\
\hline Single & $-1.67(-2.11--1.23)$ & $<0.0001 *$ \\
\hline \multicolumn{3}{|l|}{ Financial aid } \\
\hline None & Reference & Reference \\
\hline Retirement pension & $-5.66(-8.53--2.79)$ & $<0.0001 *$ \\
\hline Disability pension/early retirement & $-5.00(-5.59--4.40)$ & $<0.0001 *$ \\
\hline Social benefits & $-4.55(-5.13--3.98)$ & $<0.0001^{*}$ \\
\hline \multicolumn{3}{|l|}{ Heritage } \\
\hline Swedish-born, Swedish-descendant & Reference & Reference \\
\hline Swedish-born, non-Swedish-descendant & $-3.19(-4.89--1.50)$ & $0.0002 *$ \\
\hline Born outside Sweden & $-4.14(-4.7--3.67)$ & $<0.0001^{*}$ \\
\hline
\end{tabular}

${ }^{a}$ Adjusted for operation year, baseline OP-score, age, sex, BMI, sleep apnoea, hypertension, diabetes, dyslipidaemia, dyspepsia/GERD, depression and $\%$ EBMIL

*Significant $p$ value $(p<0.05)$ after correction for multiple calculations

difference in improvement in HRQoL may well be caused by low social support and low health literacy. Furthermore, preoperative education and postoperative follow-up programmes are usually designed to suit the majority of patients, i.e. the middle-aged, Swedish-born population with easy access to a follow-up clinic. Inability of the system to adapt to the requirements of other patient groups may result in fewer attending important follow-up visits [36].
All socioeconomic subgroups examined in this study reported significantly improved HRQoL. However, groups generally viewed as having lower socioeconomic status consistently reported less improvement in their HRQoL. It is thus obvious that this group together with younger patients, higher BMI and women require better support in the pre- and postoperative periods. Further studies are needed to identify the specific needs of these groups and at a later stage to evaluate the effects of specifically targeted interventions. 


\section{Strengths and Limitations}

The main strength of this study lies in the high number of patients included, and the very high quality of data provided by the two registers (SOReg and Statistics Sweden). The major weaknesses lie in the high proportion of patients without a self-reported HRQoL and the retrospective nature of the study. Although patients not reporting their HRQoL generally came from the groups with poorer postoperative HRQoL, the sensitivity analysis differences were small implying acceptable generalisability to all patients operated with laparoscopic gastric bypass surgery in Sweden. However, the homogeneity of the study group may limit the reproducibility in other parts of the world. Using the OP Scale to estimate HRQoL, we focused our attention on the effects of gastric bypass surgery on psychosocial aspects of HRQoL. Although this is an aspect often emphasised by patients with severe obesity [11], this itself may be a limitation since greater focus on physical or mental dimensions could have modified the results. Furthermore, the first two years after a bariatric surgical procedure is often referred to as the "honeymoon period" due to the rapid weight loss and peaking in perceived quality-of-life [23]. Although HRQoL tends to decrease to some extent after the first two years, patients generally remain more satisfied with their situation than they were prior to their operation $[12,23]$.

\section{Conclusion}

Two years after bariatric surgery, a strong association between weight loss and improvement in HRQoL was seen, though several factors influenced the degree of improvement. Younger patients with higher preoperative BMI and lower socioeconomic status showed less improvement in selfreported HRQoL after gastric bypass surgery.

Funding This work was supported by grants from Region Örebro County, Örebro University, Stockholm County Council, SRP Diabetes and the NovoNordisk Foundation. None of the supporting agents had any influence on the contents of this article.

\section{Compliance with Ethical Standards}

Conflict of Interest Ingmar Näslund received consultant fees from Baricol Bariatrics AB, Sweden; AstraZeneca AB, Sweden; and Ethicon, Johnson \& Johnson A7S, Denmark, for work unrelated to the contents of this study. The other authors declare that they have no conflict of interest.

Ethical Approval The study was conducted in accordance with the ethical standards of the 1964 Helsinki Declaration and its later amendments and with the approval of the regional ethics committee in Uppsala, Sweden.
Open Access This article is distributed under the terms of the Creative Commons Attribution 4.0 International License (http:// creativecommons.org/licenses/by/4.0/), which permits unrestricted use, distribution, and reproduction in any medium, provided you give appropriate credit to the original author(s) and the source, provide a link to the Creative Commons license, and indicate if changes were made.

\section{References}

1. Must A, Spadano J, Coakley EH, et al. The disease burden associated with overweight and obesity. JAMA. 1999;282(16):1523-9.

2. Field AE, Coakley EH, Must A, et al. Impact of overweight on the risk of developing common chronic diseases during a 10-year period. Arch Intern Med. 2001;161(13):1581-6.

3. Renehan AG, Tyson M, Egger M, et al. Body-mass index and incidence of cancer: a systematic review and meta-analysis of prospective observational studies. Lancet. 2008;371(9612):569-78.

4. Fontaine KR, Redden DT, Wang C, et al. Years of life lost due to obesity. JAMA. 2003;289(2):187-93.

5. Adams TD, Davidson LE, Litwin SE, et al. Health benefits of gastric bypass surgery after 6 years. JAMA. 2012;308(11):1122-31.

6. Sjostrom L, Narbro K, Sjostrom CD, et al. Effects of bariatric surgery on mortality in Swedish obese subjects. N Engl J Med. 2007;357(8):741-52.

7. Sjostrom L, Gummesson A, Sjostrom CD, et al. Effects of bariatric surgery on cancer incidence in obese patients in Sweden (Swedish Obese Subjects Study): a prospective, controlled intervention trial. Lancet Oncol. 2009;10(7):653-62.

8. Sundbom M, Hedberg J, Marsk R, et al. Substantial decrease in comorbidity 5 years after gastric bypass: a population-based study from the Scandinavian Obesity Surgery Registry. Ann Surg. 2017;265(6):1166-71.

9. Megias A, Gonzalez-Cutre D, Beltran-Carrillo VJ, et al. The impact of living with morbid obesity on psychological need frustration: a study with bariatric patients. Stress Health. 2018;34(4):509-22.

10. Kolotkin RL, Crosby RD, Williams GR, et al. The relationship between health-related quality of life and weight loss. Obes Res. 2001;9(9):564-71.

11. Karlsson J, Taft C, Sjostrom L, et al. Psychosocial functioning in the obese before and after weight reduction: construct validity and responsiveness of the Obesity-related Problems scale. Int J Obes Relat Metab Disord. 2003;27(5):617-30.

12. Kolotkin RL, Kim J, Davidson LE, et al. 12-year trajectory of health-related quality of life in gastric bypass patients versus comparison groups. Surg Obes Relat Dis. 2018;14(9):1359-65.

13. Raoof M, Naslund I, Rask E, et al. Health-related quality-of-life (HRQoL) on an average of 12 years after gastric bypass surgery. Obes Surg. 2015;25(7):1119-27.

14. Martin M, Beekley A, Kjorstad R, et al. Socioeconomic disparities in eligibility and access to bariatric surgery: a national populationbased analysis. Surg Obes Relat Dis. 2010;6(1):8-15.

15. Wallace AE, Young-Xu Y, Hartley D, et al. Racial, socioeconomic, and rural-urban disparities in obesity-related bariatric surgery. Obes Surg. 2010;20(10):1354-60.

16. Stenberg E, Persson C, Näslund E, et al. The impact of socioeconomic factors on the early postoperative complication rate after laparoscopic gastric bypass surgery. Surg Obes Relat Dis. 2019;15(4):575-81.

17. Courtney PM, Huddleston JI, Iorio R, et al. Socioeconomic risk adjustment models for reimbursement are necessary in primary total joint arthroplasty. J Arthroplast. 2017;32(1):1-5. 
18. Goljo E, Parasher AK, Iloreta AM, et al. Racial, ethnic, and socioeconomic disparities in pituitary surgery outcomes. Laryngoscope. 2016;126(4):808-14.

19. Olbers T, Lonroth H, Fagevik-Olsen M, et al. Laparoscopic gastric bypass: development of technique, respiratory function, and longterm outcome. Obes Surg. 2003;13(3):364-70.

20. Holm S. A simple sequentially rejective multiple test procedure. Scand J Stat. 1979;6(2):65-70.

21. Larsson U, Karlsson J, Sullivan M. Impact of overweight and obesity on health-related quality of life-a Swedish population study. Int J Obes Relat Metab Disord. 2002;26(3):417-24.

22. Dreber $\mathrm{H}$, Thorell $\mathrm{A}$, Reynisdottir $\mathrm{S}$, et al. Health-related quality of life 5 years after Roux-en-Y gastric bypass in young (18-25 years) versus older ( $>/=26$ years) adults: a Scandinavian Obesity Surgery Registry Study. Obes Surg. 2019;29(2):434-43.

23. Andersen JR, Aasprang A, Karlsen TI, et al. Health-related quality of life after bariatric surgery: a systematic review of prospective long-term studies. Surg Obes Relat Dis. 2015;11(2):466-73.

24. Puhl RM, King KM. Weight discrimination and bullying. Best Pract Res Clin Endocrinol Metab. 2013;27(2):117-27.

25. Strauss RS. Childhood obesity and self-esteem. Pediatrics. 2000;105(1):e15.

26. Kolotkin RL, Crosby RD, Williams GR. Health-related quality of life varies among obese subgroups. Obes Res. 2002;10(8):748-56.

27. Varban OA, Cassidy RB, Bonham A, et al. Factors associated with achieving a body mass index of less than 30 after bariatric surgery. JAMA Surg. 2017;152(11):1058-64.

28. Rowlands G, Protheroe J, Winkley J, et al. A mismatch between population health literacy and the complexity of health information: an observational study. Br J Gen Pract. 2015;65(635):e379-86.
29. Johnson TV, Abbasi A, Kleris RS, et al. Assessment of single-item literacy questions, age, and education level in the prediction of low health numeracy. JAAPA. 2013;26(8):50-4.

30. Kolt GS, George ES, Rebar AL, et al. Associations between quality of life and duration and frequency of physical activity and sedentary behaviour: baseline findings from the WALK 2.0 randomised controlled trial. PLoS One. 2017;12(6):e0180072.

31. Kunst AE, Bos V, Lahelma E, et al. Trends in socioeconomic inequalities in self-assessed health in 10 European countries. Int $\mathrm{J}$ Epidemiol. 2005;34(2):295-305.

32. Carden A, Blum K, Arbaugh CJ, et al. Low socioeconomic status is associated with lower weight-loss outcomes 10-years after Rouxen-Y gastric bypass. Surg Endosc. 2019;33(2):454-9.

33. Brydsten A, Hammarstrom A, San Sebastian M. Health inequalities between employed and unemployed in northern Sweden: a decomposition analysis of social determinants for mental health. Int $\mathrm{J}$ Equity Health. 2018;17(1):59.

34. Lindstrom M. Marital status, social capital, material conditions and self-rated health: a population-based study. Health Policy. 2009;93(2-3):172-9.

35. Bylund A, Benzein E, Sandgren A. Stabilizing family life after gastric bypass surgery. Int J Qual Stud Health Well Being. 2017;12(1):1325674.

36. Aarts MA, Sivapalan N, Nikzad SE, et al. Optimizing bariatric surgery multidisciplinary follow-up: a focus on patient-centered care. Obes Surg. 2017;27(3):730-6.

Publisher's Note Springer Nature remains neutral with regard to jurisdictional claims in published maps and institutional affiliations. 